\title{
The first year with the Journal of Electrical Bioimpedance (JEB)
}

\author{
Ørjan G. Martinsen and Sverre Grimnes \\ 1. Editors-in-chief, Journal of Electrical Bioimpedance \\ 2. Department of Physics, University of Oslo, Oslo, Norway \\ 3. Department of Biomedical and Clinical Engineering, Rikshospitalet, Oslo University Hospital, Oslo Norway
}

The Journal of Electrical Bioimpedance (JEB) is now one year old and it is time to look back and also revise our strategy for the future.

The general idea behind JEB was to create an open access, no publication fee, online, scientific journal for bioimpedance research. Coming from the physics field, we strongly believe in open access as the future solution for a more globally including research policy and practice. The latest results from international research should always be readily available to everyone, regardless the economy of their university or institution. Moreover, open access also increases the research impact of the individual author [1].

Physicists have a long tradition with open access; the arXiv (http://arxiv.org) was started in 1991 as a repository for preprints in physics and was later expanded to include other fields [2]. The choice of not having any publication fee has been possible only because of the willingness of many people to take this on as voluntary work. We will aim at keeping things this way as long as possible, but realize that we may need some economy when the number of submissions reach high numbers.

When we established JEB one year ago, we had an unofficial goal of publishing 5-6 articles the first year, doubling that the next year and then gradually converge towards one article per week as a young journal. We ended up publishing eight scientific papers in 2010, which we consider a good result. All of these papers have been downloaded a large number of times and the first published paper, Bernstein's review on impedance cardiography, was downloaded about 1000 times in 2010 [3].

It has also been interesting to follow the activity on the JEB site using tools like e.g. Google Analytics [4]. Two main conclusions can be drawn from this: The readers of JEB come from absolutely all over the world. (Probably from natural causes, central Africa is underrepresented, however.) The second conclusion is that the interest for JEB has been steadily increasing throughout the whole period.

Being indexed or abstracted in the large databases is of highest priority for JEB. In 2010 we became indexed in Directory of Open Access Journals (DOAJ), Index Copernicus, JournalTOCs and Open J-Gate. We will continue this process in 2011 with the very distinct goal of finally getting indexed by the Thomson ISI Web of Knowledge and getting an impact factor.
We are very fortunate to have an extremely competent group of section editors and we are very grateful for their contributions to the quality of the journal. Readers that have long experience with bioimpedance and feel that they would like to contribute to the development of JEB, are encourage to contact us.

\section{References}

1. Bernius S. The impact of open access on the management of scientific knowledge. Online Information Review. 2010;34: 583-603.

2. http://en.wikipedia.org/wiki/Arxiv (March 2, 2011)

3. Bernstein DP. Impedance cardiography: Pulsatile blood flow and the biophysical and electrodynamic basis for the stroke volume equations. J. Electr. Bioimp. 2010;1:2-17.

4. http://www.google.com/analytics (March 2, 2011) 